M. P. Mignolet, C. Soize, K. Kim, D-H Lee, Nonparametric stochastic modeling of structural dynamic and aeroelastic systems: formulation and novel extensions, 9th Non-deterministic Approaches (NDA) Conference and 48th AIAA/ASME/ASCE/AHS/ASC Structures, Structural Dynamics, and Materials (SDM) Conference, Waikiki, Hawaii, April 23-26, 2007.

\title{
NONPARAMETRIC STOCHASTIC MODELING OF STRUCTURAL DYNAMIC AND AEROELASTIC SYSTEMS: FORMULATION AND NOVEL EXTENSIONS
}

\author{
Marc P. Mignolet ${ }^{*}$ \\ Arizona State University, Tempe, AZ 85287-6106, USA \\ Christian Soize \\ Université de Marne-la-Vallée, 77454 Marne-la-Vallée, France \\ Kwangkeun $\mathrm{Kim}^{\dagger}$ \\ Arizona State University, Tempe, AZ 85287-6106, USA \\ and Dong-Hwan Lee ${ }^{* *}$ \\ ZONA Technology, Scottsdale, AZ 85258, USA
}

\begin{abstract}
This paper addresses the modeling and simulation of random structural dynamic and aeroelastic systems, linear and nonlinear, using the nonparametric methodology. The fundamental theory underlying this approach is first review and its application to linear structural dynamic models is demonstrated. Next, the theory is extended in two distinct directions: i.e. geometrically nonlinear models and an improved characterization of the natural frequencies of random linear systems.
\end{abstract}

\section{INTRODUCTION}

The stochastic modeling and simulation of random multi-degree-of-freedom systems has often in the past been accomplished by postulating joint probability density functions of the components of their mass, stiffness, and/or damping matrices or, equivalently, of their corresponding natural frequencies, damping ratios, and mode shapes (e.g. Ghanem and Spanos, 1991, Kleiber et al, 1992, Rivas-Guerra and Mignolet, 2004, Schueller, 1997). However, a different approach has recently been proposed (Soize, 2000, 2001) and validated (see Soize, 2005, for a review) in which the probabilistic model of the mass, stiffness, and/or damping matrices is not assumed but rather determined to maximize the entropy under the constraints (i) that these matrices are positive definite, (ii) that their mean values are prescribed, and (iii) of an overall measure of variation (variance of the norms of the matrices prescribed).

Professor, Department of Mechanical and Aerospace Engineering, Associate Fellow, AIAA.

* Professor, Laboratoire de Mécanique

${ }^{\dagger}$ Postdoctoral Fellow, Department of Mechanical and Aerospace Engineering.

${ }^{* *}$ R\&D Engineering Specialist, AIAA Member
This approach, which has been named nonparametric since no parameter value is selected by the user, is applicable to a broad range of applications in which little is known about the variability of the system.

The basic theory underlying the nonparametric approach is first review and its application to two aeroelastic models is demonstrated. Next, two extensions of this formulation are accomplished. The first of these extension focuses on geometrically nonlinear models, e.g. beam and plates the response of which is of the order of or larger than the thickness. This extension requires the appropriate handling of the nonlinear stiffness tensors/matrices that are present in the model.

Attention is next focused on applications in which routine testing is conducted on small samples to obtain a basic assessment of variability. Such testing typically yields measurement of the natural frequencies and estimates of their variances. In this light, the goal of the present investigation is to extend the formulation of the nonparametric approach to allow for additional constraints on the variance of some of the eigenvalues of the mass, damping, and/or stiffness matrices.

\section{ENTROPY MAXIMIZATION AND CONSTRAINTS}

It is desired here to simulate realizations of symmetric positive definite random matrices, e.g. the mass and/or the stiffness matrix of a linear dynamic system, the properties of which, i.e. eigenvalues, eigenvectors, components, etc., are all random. This requirement necessitates the specification of the joint probability density function of all elements of the matrix. In most practical problems, however, this information is not available - only some moments and/or marginal probability density functions are likely to be available. In the absence of the exact distribution, it is then 
appropriate to ask what are the desirable features of this distribution. In this context, note that the design of structural systems is often robust, i.e. that small perturbations in their geometrical and material properties do not alter significantly the probability of failure/fatigue life of the system considered (see Rivas-Guerra and Mignolet, 2004, for a notable counterexample in turbomachinery). It is thus desirable to dispose of a probabilistic model which places particular emphasis on "larger" deviations from the design conditions. Equivalently, this model should have a large value of the entropy as defined by

$$
S=-\int_{\Omega} p_{\underline{X}}(\underline{x}) \ln p_{\underline{X}}(\underline{x}) d \underline{x}
$$

where $X$ denotes the vector of random variables considered of joint probability density function $p_{\underline{X}}(\underline{x})$. Further, $\underline{x}$ denotes the realized values of $\underline{X}$, and $\Omega$ the domain of support of $p_{\underline{X}}(\underline{x})$.

Consistently with the above discussion, a probabilistic model of uncertain $n \times n$ matrices $A$, i.e. either the mass, stiffness, and/or damping matrix of a linear system, has been formulated (Soize, 2000, 2001) to maximize the value of the entropy $S$

$$
S=-\int_{\Omega} p_{\underline{A}}(\underline{\underline{a}}) \ln p_{\underline{\underline{A}}}(\underline{\underline{a}}) d \underline{\underline{a}}
$$

given the following physical constraints:

$$
\begin{gathered}
\int_{\Omega} p_{\underline{A}}(\underline{\underline{a}}) d \underline{\underline{a}}=1 \\
\left.E[\underline{\underline{A}}]=\int_{\Omega} \underline{\underline{a}} \underline{\underline{\underline{a}}} p_{\underline{\underline{A}}} \underline{\underline{\underline{a}}}\right) d \underline{\underline{a}}=\underline{\underline{A}}
\end{gathered}
$$

and

$$
\int_{\Omega} \ln [\operatorname{det}(\underline{\underline{a}})] p_{\underline{\underline{A}}}(\underline{\underline{a}}) d \underline{\underline{a}}=v \text { finite }
$$

where $E[$.$] denotes the operation of mathematical$ expectation and $\operatorname{det}(\underline{=})$ is the determinant of $\underline{=}$.

The first two of the above constraints correspond to the normalization of the total probability to 1 (Eq. (3)) and the specification of the mean matrix (Eq. (4)). The third one, Eq. (5), implies the existence of the mean squared Frobenius norm of the inverse matrix $\underline{\underline{A}}^{-1}$ (see Soize $(2000,2001)$ for discussion). To apply this approach to the simulation of random mass, stiffness, and damping matrices of dynamical systems, it is further required to ensure both the symmetry and positive definiteness of every realized matrix $\underset{=}{A}$. This is achieved by introducing the Cholesky decomposition of $\underline{A}$, i.e.

$$
\underline{\underline{A}}=\underline{\underline{\tilde{L}}} \underline{\underline{L}}^{T}
$$

where $\underline{\underline{L}}$ is an lower triangular matrix with nonnegative diagonal elements and ${ }^{T}$ denotes the operation of matrix transposition. The domain of support $\Omega$ of the obtained probability density function is then such that the elements $\widetilde{L}_{i j}$ belong to $(-\infty,+\infty)$ for $i \neq j$ and $[0,+\infty)$ for $i=j$, i.e.

$$
\begin{aligned}
\Omega=\left\{\underline{\underline{a}}=\underline{\underline{L}} \underline{\underline{L}}^{T} ; \widetilde{L}_{i j}, i, j=1, \ldots, n:\right. \\
\\
{\left.\left[\widetilde{L}_{i j} \in(-\infty,+\infty), i>j\right] \cap\left[\widetilde{L}_{i i} \in[0,+\infty)\right]\right\} . }
\end{aligned}
$$

Not only have the theoretical aspects of this formulation been discussed extensively but it also been broadly validated and applied (see Soize (2005) for a review). Note in the above approach that the level of uncertainty is controlled by the single parameter $v$ so that only a broad knowledge of the matrix uncertainty needs to be known.

\section{FUNDAMENTAL PROBABILISTIC MODEL}

\section{Derivation}

Following the discussions of the previous sections, the proposed probabilistic model $p_{\underline{\underline{A}}}(\underline{\underline{a}})$ maximizes the entropy, $S$ of Eq. (2), under the constraints of Eqs (3)(5) as well as the symmetry and positive definiteness requirement of Eq. (6). Using Lagrange multipliers $\mu_{0}$, $\underline{\underline{\mu}}$, and $\lambda-1$ the constrained maximization of Eq. (2) is reduced to the unconstrained maximization of

$$
\begin{gathered}
\left.S^{*}=S-\mu_{0} \int_{\Omega} p_{\underline{A}}(\underline{\underline{a}}) d \underline{\underline{a}}-\operatorname{tr}\left\{\underline{\tilde{\mu}}^{T} \int_{\Omega} \underline{\underline{a}} \underline{p}_{\underline{A}} \underline{\underline{a}} \underline{\underline{a}}\right) d \underline{\underline{a}}\right\} \\
\left.+(\lambda-1) \int_{\Omega} \ln [\operatorname{det}(\underline{\underline{a}})] p_{\underline{\underline{A}}} \underline{\underline{a}} \underline{\underline{a}}\right) d \underline{\underline{a}}
\end{gathered}
$$

Proceeding next by calculus of variations, it is shown that

$$
p_{\underline{\underline{A}}}(\underline{\underline{a}})=\widetilde{C}[\operatorname{det}(\underline{\underline{a}})]^{\lambda-1} \exp \left[-\operatorname{tr}\left(\underline{\underline{\mu}}^{T} \underline{\underline{a}}\right)\right]
$$

where $\widetilde{C}$ is the appropriate normalization constant to satisfy the normalization condition, Eq. (3). Before addressing the evaluation of the Lagrange multipliers, it is desired to simplify Eq. (8) and to address the positive definiteness requirement. In regards to simplifications, introduce first the matrix $\underline{\underline{L}}$ such that

$$
\underline{\underline{A}}=\underline{\underline{L}} \underline{\underline{L}} \underline{\underline{L}}
$$

where $\underline{\underline{L}}$ is any decomposition, e.g. Cholesky, of $\overline{\underline{A}}$. Next, express the random matrix $\underset{=}{=}$ as

$$
\underline{\underline{A}}=\underline{\underline{L}} \underline{\underline{G}} \underline{\underline{L}}^{T}
$$


Proceeding with this change of random variables, it is found that the probability density function of the elements of $\underline{G}$ is

$$
\left.\left.p_{\underline{\underline{G}}}^{\underline{\underline{(g}}} \underline{\underline{\underline{G}}}\right)=\bar{C} \mid \operatorname{det}(\underline{\underline{g}})\right]^{\lambda-1} \exp [-\operatorname{tr}(\underline{\underline{\mu}} \underline{\underline{g}} \underline{\underline{g}}) \mid
$$

where $\bar{C}$ is a new normalization constant and $\underline{\underline{\mu}}=\underline{\underline{L}} \underline{\underline{\mu}} \underline{\underline{L}}$.

To guarantee the symmetry and positive definiteness of $\underline{\underline{G}}$, and thus of $\underline{A}$, the model of Eq. (11) is reformulated in terms of the elements of the lower triangular matrix $\underline{\underline{H}}$ such that

$$
\underline{\underline{G}}=\underline{\underline{H}} \underline{\underline{H}}^{T} \text {. }
$$

In terms of elements, the above matrix equality can be rewritten as

$$
G_{i j}=\sum_{k=1}^{j} H_{i k} H_{j k} \quad j \leq i
$$

or

$$
\begin{gathered}
G_{i 1}=H_{i 1} H_{11} \quad 1<i \\
G_{i j}=H_{i j} H_{j j}+\sum_{k=1}^{j-1} H_{i k} H_{j k} \quad 1<j<i
\end{gathered}
$$

and

$$
\begin{gathered}
G_{11}=H_{11}^{2} \\
G_{i i}=H_{i i}^{2}+\sum_{k=1}^{i-1} H_{i k}^{2} \quad 1<i
\end{gathered}
$$

As demonstrated in Soize (2000), the Jacobian of the transformation is

$$
J=2^{n} \prod_{l=1}^{n} h_{l l}^{n-l+1}
$$

so that

$$
\left.p_{\underline{\underline{H}}} \underline{\underline{h}}\right)=C\left\{\prod_{l=1}^{n} h_{l l}^{n-l+2 \lambda-1}\right\} \exp \left[-\operatorname{tr}\left(\underline{\underline{h}}^{T} \underline{\mu}^{T} \underline{\underline{h}}\right)\right]
$$

where $C$ is the appropriate normalization constant over the domain

$$
\bar{\Omega}=\left\{h_{i j}, i, j=1, \ldots, n:\left[h_{i j} \in(-\infty,+\infty), i>j\right] \cap\left[h_{i i} \in[0,+\infty)\right]\right\} .
$$

To evaluate the Lagrange multipliers $\mu$, it is necessary to first express the constraint of Eq. (4) in terms of the elements of the random matrix $\underline{H}$. Combining Eqs (4), (10), and (12), it is found that

$$
E \underline{\underline{G}}\rfloor=\underline{\underline{I}}
$$

where $I_{=n}$ denotes the $n \times n$ identity matrix. The simplicity of this condition implies an equally simple form of the matrix $\underline{\mu}$. Specifically, it will be shown that this matrix is diagonal. Under this assumption, Eq. (19) reduces to

$$
\begin{aligned}
p_{\underline{\underline{H}}}(\underline{\underline{h}})=\prod_{i=1}^{n}\left\{C_{i} h_{i i}^{p(i)} \exp \left[-\mu_{i i} h_{i i}^{2}\right]\right\} \\
\times \prod_{i=1}^{n} \prod_{l=1}^{i-1}\left\{C_{i l} \exp \left[-\mu_{i i} h_{i l}^{2}\right]\right\}
\end{aligned}
$$

where $C_{i}, i=1, \ldots, n$, and $C_{i l}, i=1, \ldots, n ; l=1, \ldots, i-1$, are appropriate normalization constants and

$$
p(i)=n-i+2 \lambda-1 \text {. }
$$

It is concluded from Eq. (21) that:

(i) the elements $H_{i l}, i>l$, are all independent of each other and independent of the other elements $H_{i l}$. Further, they are normally distributed with mean 0 and standard deviation $\sigma_{i l}=1 / \sqrt{2 \mu_{i i}}$.

(ii) the elements $H_{i i}$ are all independent of each other and independent of the other elements $H_{i l}$. Further, they are distributed according to

$$
p_{H_{i i}}\left(h_{i i}\right)=C_{i} h_{i i}^{p(i)} \exp \left\lfloor-\mu_{i i} h_{i i}^{2}\right\rfloor, h_{i i} \geq 0
$$

where

$$
C_{i}=\frac{2 \mu_{i i}^{[p(i)+1] / 2}}{\Gamma((p(i)+1) / 2)}
$$

and $\Gamma($.$) denotes the Gamma function.$

From the first observation, (i), it is concluded that $E\left[G_{i l}\right]=0$ for $i \neq l$. It then remains to satisfy the diagonal terms of the mean condition, Eq. (20). To this end, note that

$$
E\left[G_{i i}\right]=1=\sum_{l=1}^{i} E\left[H_{i l}^{2}\right]=(i-1) \frac{1}{2 \mu_{i i}}+E\left[H_{i i}^{2}\right]
$$

where $E\left[H_{i i}^{2}\right]$ is obtained by integration of Eq. (23) as $(p(i)+1) / 2 \mu_{i i}$. Combining this result and Eqs (24) and $(25)$, it is found that

$$
\mu_{i i}=\frac{n+2 \lambda-1}{2} .
$$

The generation of samples of $H_{i i}$ is simplified by considering the variable $Y_{i i}=\mu_{i i} H_{i i}^{2}$. Proceeding with the change of variables, it is found that the probability density function of $Y_{i i}$ is

$$
p_{Y_{i i}}\left(y_{i i}\right)=\frac{y_{i i}^{(p(i)-1) / 2}}{\Gamma[(p(i)+1) / 2]} \exp \left[-y_{i i}\right], y_{i i} \geq 0
$$

Accordingly, it is found that $Y_{i i}$ is a Gamma distributed random variable for which efficient simulation algorithms exist, e.g. see Devroye (1986). Once a sample of $Y_{i i}$ has been simulated according to the Gamma distribution, the corresponding value of $H_{i i}, i$ $>m$, is found as

$$
H_{i i}=\sqrt{\frac{Y_{i i}}{\mu_{i i}}}
$$




\section{Step-By-Step Approach}

The simulation of random matrices $A$ according to the model derived above is achieved as follows.

(1) The parameter $\lambda$ is selected (at least tentatively)

(2) The mean model matrix $\bar{A}$ is decomposed by Cholesky factorization, see Eq.(9), and $\overline{\underline{L}}$ is obtained.

(3) $\mu_{i i}=\frac{n+2 \lambda-1}{2}$, Eq. (26), and $\sigma_{i l}=1 / \sqrt{2 \mu_{i i}}$

(4) The elements $H_{i l}, i>l$, of a lower triangular random matrix $\underline{\underline{H}}$ are generated as independent Gaussian random variables with mean zero and standard deviation $\sigma_{i l}$.

(5) For each value of $i=1, \ldots, n$, a Gamma distributed random variable $Y_{i i}$ of parameter $(p(i)+1) / 2=$ $(n-i+2 \lambda) / 2$ is simulated.

(6) For each value of $i=1, \ldots, n$, the diagonal element $H_{i i}$ of the lower triangular random matrix $\underline{\underline{H}}$ is determined as $H_{i i}=\sqrt{Y_{i i} / \mu_{i i}}$.

(7) The random matrix $\stackrel{A}{=}$ is obtained as $\underline{\underline{A}}=\underline{\underline{L}} \underline{\underline{H}} \underline{\underline{H}}^{T} \underline{\underline{L}}^{T}$, Eq. (10) and (12).

(8) Steps (4)-(7) are repeated as many time as necessary to produce the population of random matrices $\underset{\underline{A}}{=}$.

(9) The parameter $\lambda$ is adjusted if necessary (for example if this parameter is prescribed by the standard deviation of an eigenvalue).

\section{Examples of Application}

The above concepts will be validated here on an aeroelastic system, namely the Goland wing of Fig. 1 (see Table 1 for natural frequencies). The effects of uncertainty in the structural properties (more specifically on the stiffness matrix) on the aeroelastic response, i.e. flutter boundary and forced response, will be assessed.

A flutter analysis conducted with ZAERO with a 20 mode model demonstrated that the Goland wing (referred later on to the mean flutter wing) flutters at $646.24 \mathrm{ft} / \mathrm{s}$ with a frequency of $1.958 \mathrm{~Hz}$.

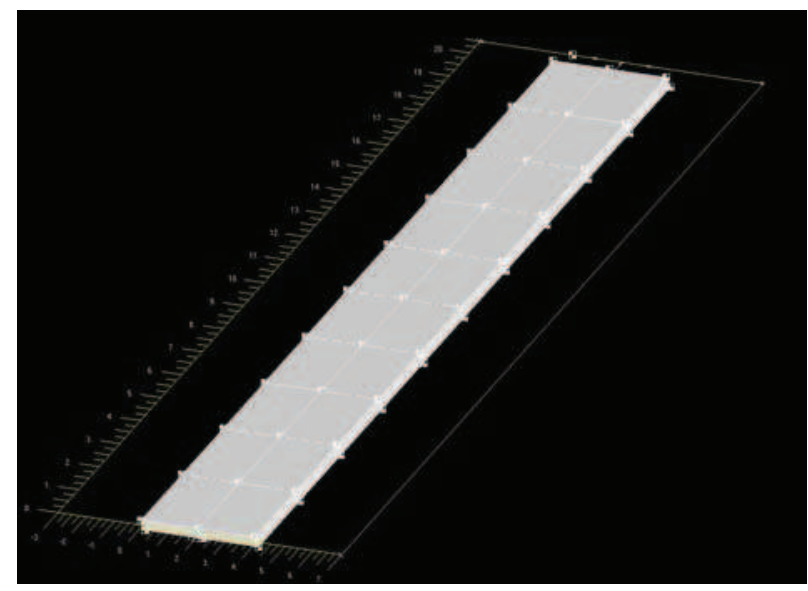

Figure 1. The Goland wing model.

\begin{tabular}{|c|c|c|c|}
\hline Mode \# & $\begin{array}{c}\text { Nat. Freq. } \\
(\mathrm{Hz})\end{array}$ & Mode \# & $\begin{array}{c}\text { Nat. Freq. } \\
(\mathrm{Hz})\end{array}$ \\
\hline 1 & 1.690 & 6 & 16.260 \\
\hline 2 & 3.051 & 7 & 22.845 \\
\hline 3 & 9.172 & 8 & 26.318 \\
\hline 4 & 10.834 & 9 & 29.183 \\
\hline 5 & 11.258 & \multicolumn{2}{|c}{} \\
\cline { 2 - 3 } & &
\end{tabular}

Table 1. Natural frequencies of the mean Goland wing

It was desired to assess the variations of the flutter speed and flutter frequency that would result from a variation of the natural frequencies of the wing. To this end, the nonparametric approach was used with the 20 mode reduced order model and the parameter $\lambda$ was selected so that the random Goland wings would exhibit a standard deviation of the first natural frequency equal to $1 \%$ of its value for the mean model (i.e. $1.690 \mathrm{~Hz}$ ). An ensemble of 300 such stiffness matrices were generated and for each matrix, the flutter speed and frequency was recomputed by ZAERO. A shotgun plot of the results is presented in Fig. 2.

While most of the random wings generated fluttered in conditions near those of the mean Goland wing (the blue dots in Fig. 2(a)), 4 of them did not. More specifically, these 4 outliers exhibited much higher flutter frequencies and flutter speeds as if the flutter in the low order modes ( 1 and 2 which are bending and torsion, respectively) had been prevented. Since the flutter mechanism of the Goland wing is based on the aerodynamic coupling of the first bending and torsion modes, it appeared that the coupling that is induced between these modes by the uncertainty would be a key parameter. Accordingly, the 300 values of the flutter 


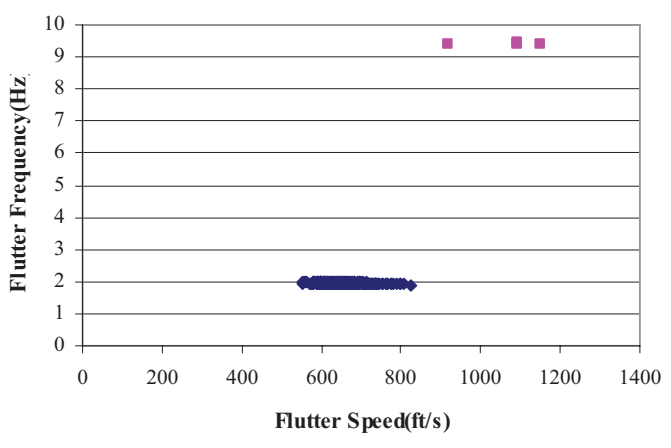

(a)

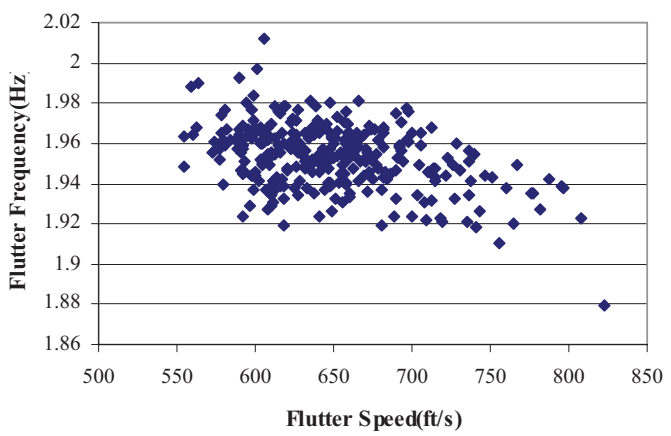

(b)

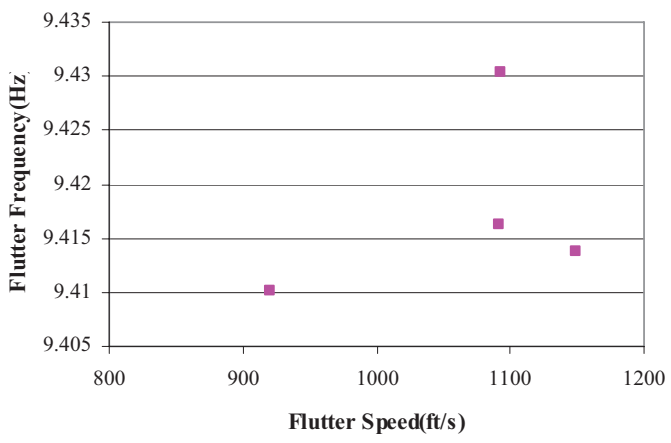

(c)

Figure 2. Shotgun plot of the flutter frequency vs. flutter speed for the 300 random Goland wings (a) all 300 results, (b) and (c) zoomed.

speed were plotted again but vs. the random stiffness coefficient $K_{12}$, see Fig. 3. This figure suggests that there exists a threshold value of $K_{12}$ beyond which the aerodynamic will not be able to induce flutter in the first two modes and that the 4 outliers had values of $K_{12}$ exceeding this threshold. Accordingly, these 4 wings were found to flutter through the coupling of modes 3 and 4, see Fig. 4.

Having justified the occurrence of the 4 peculiar cases,

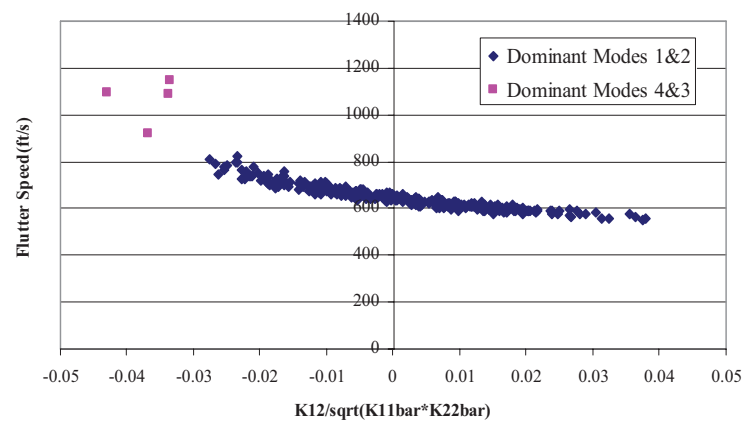

Figure 3. Shotgun plot of the flutter speed vs. normalized stiffness coefficient $K_{12}$

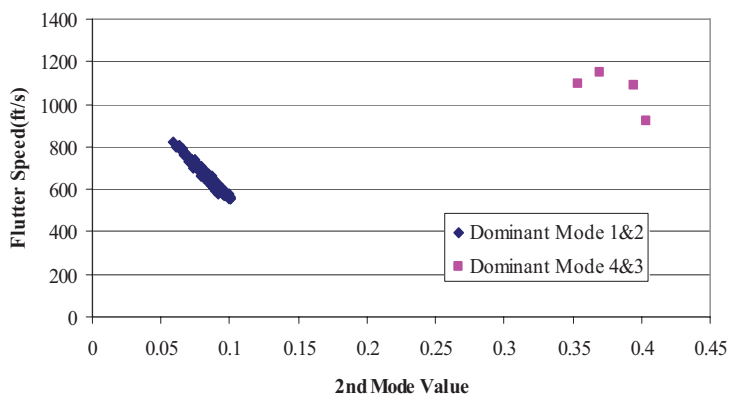

Figure 4. Shotgun plot of the flutter speed vs. the second highest component of the normalized flutter mode

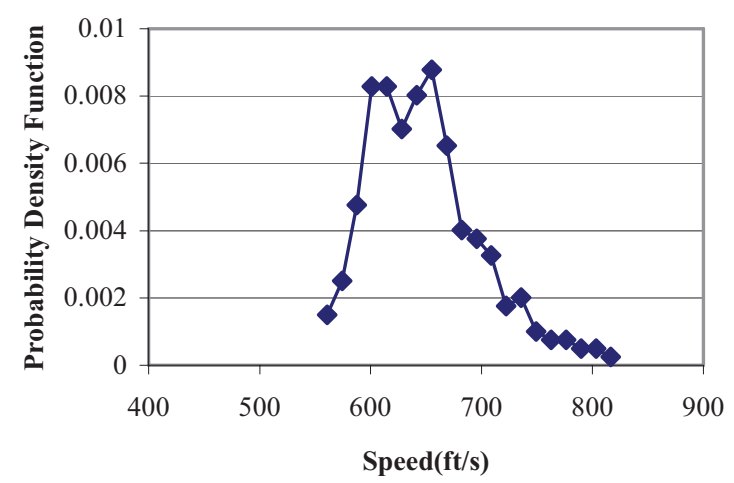

Figure 5. Probability density function of the flutter speed.

the remaining 296 flutter instances were analyzed and the probability density functions of the flutter speed and frequency were estimated, see Fig. 5-6. It is seen from these figures that the distribution of the flutter speed is particularly skewed with a higher emphasis on the high tail. Accordingly, the effects of uncertainty will often be positive, i.e. leading to an increase in the flutter speed. 


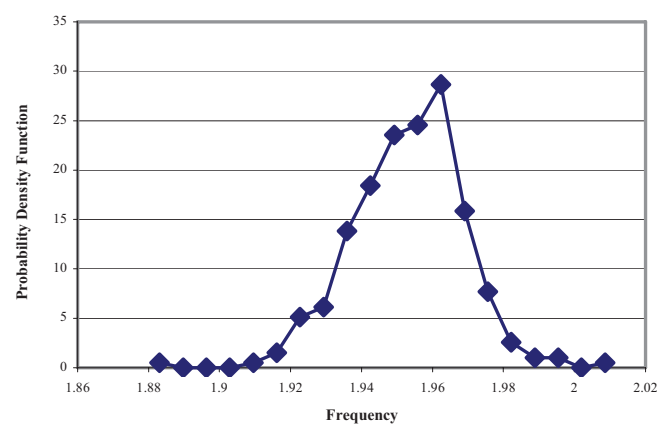

Figure 6. Probability density function of the flutter frequency.

\section{EXTENSION TO GEOMETRICALLY NONLINEAR STRUCTURES}

The extension of the fundamental nonparametric model discussed above to geometrically nonlinear structural dynamics model requires first the availability of a dynamic model for the system. Adopting an elastic material model in which the second Piola-Kirchhoff stress tensor is linearly related to the Green strain tensor, it is found that the governing equations of a structures are governed by equations of the type (e.g. see Mignolet and Soize, 2007)

$$
\underline{\underline{\hat{M}}}+\underline{\underline{\hat{C}}} \underline{\underline{\alpha}}+\underline{\hat{K}}_{N L}=\underline{F}(t)
$$

where $\underline{\hat{K}}_{N L}$ is the vector of nonlinear restoring forces the elements of which can be expressed as

$$
\begin{array}{r}
\left(\underline{\hat{K}}_{N L}\right)_{i}=\sum_{j=1}^{N} \hat{K}_{i j}^{(1)} x_{j}+\sum_{j, l=1}^{N} \hat{K}_{i l j}^{(2)} x_{l} x_{j} \\
+\sum_{j, l, p=1}^{N} \hat{K}_{i l j p}^{(3)} x_{l} x_{j} x_{p}
\end{array}
$$

where $N$ is the total number of degrees-of-freedom in the finite element model. Further, every stress component $S$ at every point of the panel can be written in the form

$$
S=\hat{S}^{(0)}+\sum_{i=1}^{N} \hat{S}_{i}^{(1)} x_{l}+\sum_{i, j=1}^{N} \hat{S}_{i j}^{(2)} x_{i} x_{j} .
$$

The nonparametric stochastic modeling approach is best applied to reduced order model. Then, proceeding with a modal representation truncated to $m$ modes leads to reduced order model governing equations that exhibit the same cubic nonlinearity as in Eq. (30), i.e.

$$
\underline{\underline{M}} \underline{\underline{C}} \underline{\underline{\Phi}}+\underline{\bar{K}}_{N L}=\underline{\bar{F}}(t)
$$

where

$$
\begin{array}{r}
\left(\underline{\bar{K}}_{N L}\right)_{i}=\sum_{j=1}^{m} \bar{K}_{i j}^{(1)} q_{j}+\sum_{j, l=1}^{m} \bar{K}_{i l j}^{(2)} q_{l} q_{j} \\
+\sum_{j, l, p=1}^{m} \bar{K}_{i l j p}^{(3)} q_{l} q_{j} q_{p}
\end{array}
$$

and

$$
S=\hat{S}^{(0)}+\sum_{i=1}^{m} \bar{S}_{i}^{(1)} q_{l}+\sum_{i, j=1}^{m} \bar{S}_{i j}^{(2)} q_{i} q_{j}
$$

The next step in the application of the nonparametric methodology is the statement of the constraints, e.g. positive definiteness, that exist between the different stiffness terms. In fact, this constraint is that the matrix

$$
\overline{\underline{K}}_{B}=\left[\begin{array}{ll}
\overline{\bar{K}}^{(1)} & \underline{\underline{\hat{K}}} \\
\overline{\hat{\hat{K}}}^{(2) T} & 2 \underline{\underline{\underline{K}}}^{(3)}
\end{array}\right]
$$

be positive definite (see Mignolet and Soize, 2007, for a proof). In this equation, the 4 th order tensor $\bar{K}_{i l j p}^{(3)}$ has been reshaped as a square matrix $\widetilde{K}_{I J}^{(3)}$ where the indices $I$ and $J$ are $\quad I=i+(l-1) m$ and $J=j+(p-1) m$. Next, the third order tensor $\hat{K}_{i l j}^{(2)}$ is extracted from the equation

$$
\bar{K}_{i l j}^{(2)}=\frac{1}{2}\left[\hat{K}_{i l j}^{(2)}+\hat{K}_{l j i}^{(2)}+\hat{K}_{j i l}^{(2)}\right]
$$

and is further reshaped as $\hat{K}_{i l j}^{(2)}=\tilde{\hat{K}}_{i J}^{(2)}$.

The above procedure was exemplified to a beam and the reduced order modeling described by Mignolet et al. (2003) and Radu et al. (2004) was applied to determine the various stiffness coefficients in Eq. (33) and (34). Specifically, a 22 mode model consisting of 12 in-plane modes and 10 transverse modes was selected. The nonparametric approach was applied to the simulation of random matrices $\underline{\underline{K}}_{B}$ from which random linear, quadratic, and cubic stiffness coefficients $K_{i j}^{(1)}, K_{i l j}^{(2)}$, and $K_{i l j p}^{(3)}$ could be extracted. For each realization, the random equivalent of Eq. (32) and (33), i.e.

$$
\underline{\bar{M}} \underline{q}^{+} \underline{\underline{C}} \underline{\&}+\underline{K}_{N L}=\underline{\bar{F}}(t)
$$

where

$$
\begin{array}{r}
\left(\underline{K}_{N L}\right)_{i}=\sum_{j=1}^{m} K_{i j}^{(1)} Q_{j}+\sum_{j, l=1}^{m} K_{i l j}^{(2)} Q_{l} Q_{j} \\
+\sum_{j, l, p=1}^{m} \bar{K}_{i l j p}^{(3)} Q_{l} Q_{j} Q_{p}
\end{array}
$$

were solved as a function of time. Postprocessing of this data in the frequency domain then lead to the response spectra of Figs 9 and 10 for the displacement 


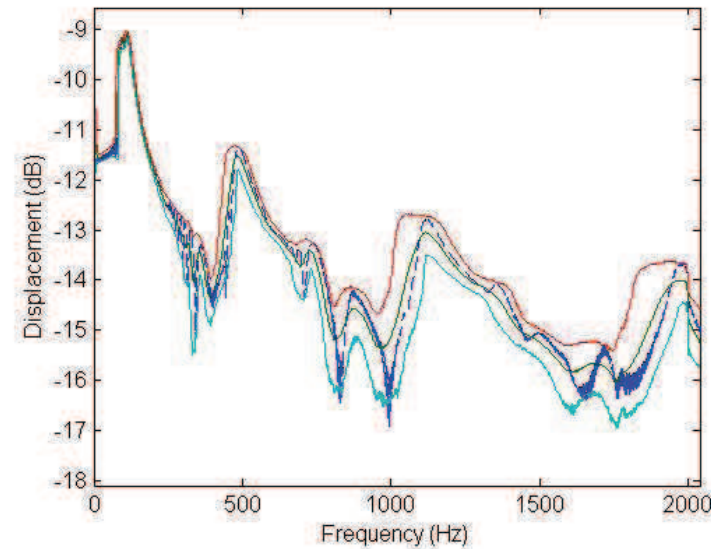

Figure 7. Transverse displacement of the middle of the random beam as a function of frequency. Mean, 5th, and 95th percentile of the spectrum and spectrum of the mean model (dashed lines).

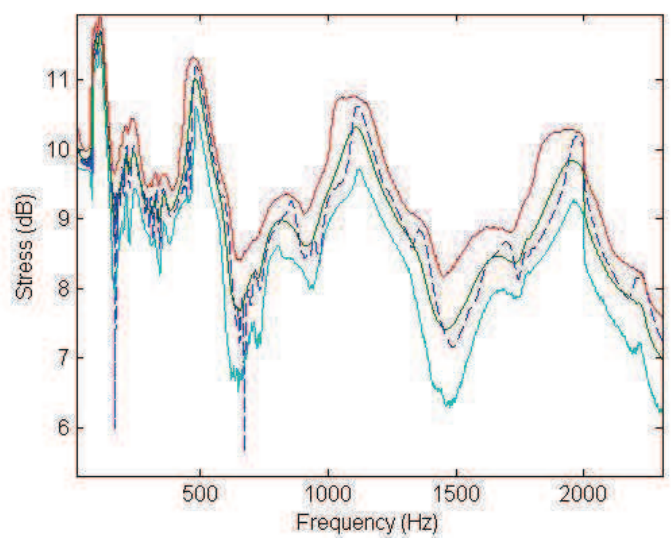

Figure 8. Stress at the middle of the random beam as a function of frequency. Mean, 5th, and 95th percentile of the spectrum and spectrum of the mean model (dashed lines).

of the beam center and the corresponding stress, as determined from

$$
S=\hat{S}^{(0)}+\sum_{i=1}^{m} \bar{S}_{i}^{(1)} Q_{l}+\sum_{i, j=1}^{m} \bar{S}_{i j}^{(2)} Q_{i} Q_{j}
$$

The consideration of temperature effects is often carried out in connection with nonlinear geometric plate/beam model. To apply the nonparametric approach to such situations, it should be noted that temperature affect only the linear stiffness terms of the where $T$ is the applied temperature differential, $\underline{\underline{K}}_{0}^{(1)}$ is the linear stiffness matrix without the temperature, and $\underline{\underline{K}}_{T}^{(1)}$ is the stiffness matrix variation induced by a unit temperature difference. In such cases, the matrix $\underline{\underline{K}}_{B 0}$ formed with $\underline{\underline{K}}_{0}^{(1)}$ instead of $\underline{\underline{K}}^{(1)}$ is indeed positive

\section{CONSIDERATION OF INFORMATION ON MULTIPLE NATURAL FREQUENCIES}

\section{Formulation}

As stated in the introduction, there are instances in which information is available to characterize the uncertainty in the structural model, e.g. variance on the lowest few natural frequencies. Then, it would be desirable to dispose of a nonparametric model that can fit the measured data. To address this extension, consider the generalized mean and random eigenvalue problems

and

$$
\underline{\underline{A}} \underline{\varphi}_{i}=\lambda_{i} \underline{\underline{B}}_{\underline{i}}
$$

$$
\underline{\underline{A}} \underline{\phi}_{i}=\tilde{\lambda}_{i} \underline{\underline{B}} \underline{\phi}_{i}
$$

where $\underline{\underline{B}}$ is a deterministic symmetric, positive definite matrix. In the ensuing discussions, it will be assumed that the eigenvectors $\underline{\varphi}_{i}$ and $\underline{\phi}_{i}$ are normalized with respect to $\underline{\underline{B}}$ so that

$$
\underline{\varphi}_{i}^{T} \underline{\underline{B}} \underline{\varphi}_{i}=1 \quad \underline{\varphi}_{i}^{T} \underline{\underline{A}}_{\underline{i}}=\underline{\lambda}_{i}
$$

and

$$
\underline{\phi}_{i}^{T} \underline{\underline{B}} \underline{\phi}_{i}=1 \quad \underline{\phi}_{i}^{T} \stackrel{\bar{A}}{=} \underline{\phi}_{i}=\tilde{\lambda}_{i}
$$

Constraining the variance of the eigenvalues $\lambda_{i}$ directly is unfortunately extremely challenging because of the lack of an exact expression for the natural frequencies of the random matrix $A$. Accordingly, an indirect approach will be selected here which relies on simple constraints that are akin to the second order moments of the eigenvalues. More specifically, it will be assumed that

$$
E\left[\left(\underline{\phi}_{i}^{T} \underline{\underline{A}} \underline{\phi}_{i}\right)^{2}\right]=\int_{\Omega}\left(\underline{\phi}_{i}^{T} \underline{\underline{a}} \underline{\phi}_{i}\right)^{2} \underline{p}_{\underline{A}}(\underline{\underline{a}}) d \underline{\underline{a}}=s_{i}^{2} \tilde{\lambda}_{i}^{2}
$$

where $s_{i}, i \in I \subseteq[1, n]$, are $m$ known positive constants and $\underline{\phi}_{i}$ are the eigenvectors of the mean matrix $\stackrel{\bar{A}}{=}$ corresponding to the $m$ eigenvalues of which the variance is known. For example, if the variances of the three lowest eigenvalues of $A$ have been estimated, then $\underline{\phi}_{i}$ will similarly be the eigenvectors of the mean matrix $\bar{A}$ corresponding to its three lowest eigenvalues. Several comments can made in regards to the constraints of Eq. (44). First, these conditions involve second order moments, not variances, but this switch is appropriate because the mean values of $\left(\phi_{i}^{T} \underline{\underline{A}} \underline{\phi}_{i}\right)$ are already prescribed by Eq. (4). Next, as discussed above, these conditions do not generally relate exactly to the natural frequencies of the random matrix $\stackrel{A}{=}$, but they do so when its eigenvectors are the same as those of its 
mean $\bar{A}$. Finally, it should be noted that the specification of the constraints of Eq. (11) provides freedom in the probabilistic model of the random matrix $A$ which can be used to match the known variances of the natural frequencies (or any other pertinent information).

The nonparametric modeling formulation for this new problem initially follows the derivation of section 3 but with some modifications. For example, the Cholesky decomposition of Eq. (9) must be rewritten as

$$
\underline{\underline{A}}=\underline{\underline{L}} \underline{\underline{L}}^{T} \quad \text { with } \quad \underline{\underline{L}}^{T} \underline{\underline{\Phi}}=\left[\begin{array}{l}
\underline{\underline{\Lambda}}^{1 / 2} \\
\underline{\underline{0}}
\end{array}\right]
$$

where $\underline{\Phi}$ denotes the $n \times m$ matrix formed by the $m$ eigenvectors $\underline{\phi}_{i}, i \in I$, and $\underline{\underline{\Lambda}}$ is the corresponding diagonal matrix of eigenvalues. Note that the condition of Eq. (45b) is introduced to simplify the constraints of Eq. (44). Note further that $\overline{\underline{L}}$ can be expressed in the partitioned form

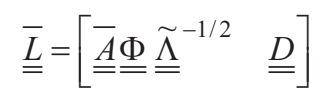

where the $n \mathrm{x}(n-m)$ matrix $\underline{\underline{D}}$ is any decomposition, e.g. Cholesky, of

$$
\underline{\underline{D}} \underline{\underline{D}}^{T}=\underline{\underline{\bar{A}}}-\underline{\underline{\bar{A}}} \underline{\underline{\Phi}} \underline{\underline{\Lambda}}^{-1} \underline{\underline{\Phi}}^{T} \underline{\underline{A}}
$$

Pre- and postmultiplying Eq. (16) by $\underline{\underline{\Phi}}^{T}$ and $\underline{\underline{\Phi}}$, respectively, it is found that $\underline{\underline{D}}^{T} \underline{\underline{\Phi}}=\underline{\underline{0}}$ as required in Eq. (45b). Following earlier steps, it is now found that the joint probability density function of the elements of the lower triangular matrix $\underline{H}$ is

$$
\begin{aligned}
p_{\underline{\underline{H}}}(\underline{\underline{h}})= & C\left\{\prod_{l=1}^{n} h_{l l}^{n-l+2 \lambda-1}\right\} \\
& \times \exp \left[-\operatorname{tr}\left(\underline{\underline{h}}^{T} \underline{\underline{\mu}}^{T} \underline{\underline{h}}\right)-\sum_{i=1}^{m} \tau_{i}\left(\sum_{l=1}^{i} h_{i l}^{2}\right)^{2}\right]
\end{aligned}
$$

where $C$ is the appropriate normalization constant over $\Omega$.

It is again found that the matrix $\underline{\underline{\mu}}$ that satisfy the mean value constraint of Eq. (4) is diagonal and the resulting joint probability density function of the elements of the lower triangular matrix $\underline{\underline{H}}$ is

$$
\begin{gathered}
\left.p_{\underline{\underline{H}}} \underline{\underline{h}} \underline{\underline{h}}\right)=\prod_{i=1}^{m}\left\{C_{i} h_{i i}^{p(i)} \exp \left[-\bar{\mu}_{i i}\left(\sum_{l=1}^{i} h_{i l}^{2}\right)-\bar{\tau}_{i}\left(\sum_{l=1}^{i} h_{i l}^{2}\right)^{2}\right]\right\} \\
\times \prod_{i=m+1}^{n}\left\{C_{i} h_{i i}^{p(i)} \exp \left[-\mu_{i i} h_{i i}^{2}\right]\right\} \times \\
\prod_{i=m+1}^{n} \prod_{l=1}^{i-1}\left\{C_{i l} \exp \left[-\mu_{i i} h_{i l}^{2}\right]\right\}
\end{gathered}
$$

where $C_{i}, i=1, \ldots, n$, and $C_{i l}, i=m+1, \ldots, n ; l=1, \ldots, i-1$, are appropriate normalization constants. This model is more complex than the original one because of the correlation that exists between some of the elements $H_{i l}$. Notwithstanding this correlation, an efficient simulation of these elements can be carried out (see details and discussion in Mignolet and Soize (2006)).

\section{Example of Application}

To exemplify the above developments, a $n=5$ degree of freedom dynamic system was considered exhibiting variations of its stiffness matrix. The above methodology was then applied with $\underline{\underline{A}}$ and $\underline{\underline{B}}$ being the stiffness and mass matrices, respectively. Further, the analysis of the system was carried out in the modal coordinates of the mean model with mass normalized modes. Thus, $\underline{\underline{B}}$ is the $5 \times 5$ unit matrix and $\underline{\underline{A}}$ is the diagonal matrix containing the squares of the natural frequencies which were first assumed to be 1, 3, 5, 7, and $9 \mathrm{rad} / \mathrm{s}$ (mean model 1). Finally, the damping matrix was constructed by assuming a damping ratio of $1 \%$ on all modes.

Three sets of computations were carried out, two with the unconstrained nonparametric approach and one with the present constrained formulation. The first unconstrained model was obtained by enforcing a standard deviation of the first natural frequency equal to 0.058 , i.e. $5.8 \%$ of the corresponding value for the mean model. The second unconstrained model was similarly determined but with a lower standard deviation, i.e. 0.033. It was observed that the ratios of the standard deviations of the natural frequencies of the random systems divided by their corresponding values for the mean models varied very little, from 0.00575 to 0.00584 for the first system and from 0.00331 to 0.00334 for the second one.

The present, constrained approach was applied next by imposing ratios of standard deviations to mean model value of $5.8 \%$ and $3.3 \%$ for the first and second natural frequency, respectively. Since the first natural frequency exhibits the largest relative variations, the first row of $\underline{H}$, i.e. the one most closely associated with the first frequency, was characterized by an unconstrained model, i.e. $\tau_{1}=0$. Given the lack of information on the third, fourth, and fifth natural frequencies, the parameters $\mu_{i i}$ and $\tau_{i}$ were assumed 
to be the same for $i \geq 2$. Accordingly, there were only 4 model parameters to be determined: $\mu_{11}, \lambda, \mu_{22}$, and $\tau_{2}$. They were iteratively obtained by imposing the prescribed standard deviations on natural frequencies (the exact constraints, not the approximate ones of Eq. (44)) and Eq. (20). A sample of 10,000 random matrices was assumed for all computations.

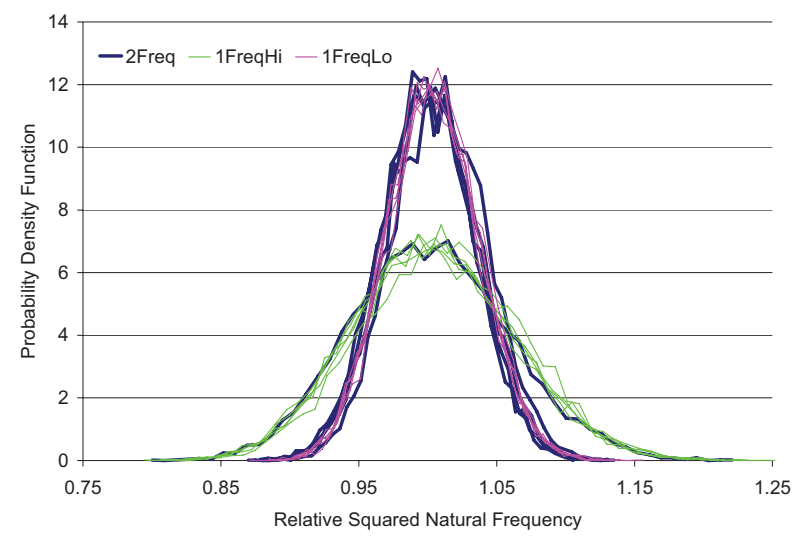

Figure 9. Probability density functions of the squared natural frequencies divided by their corresponding value for the mean model. Mean model 1. Constrained Model ("2Freq"), unconstrained models with higher

("1FreqHi") and lower ("1FreqLo") level of variations.

Shown in Fig. 9 are, for each of the three random systems, the distributions of the five natural frequencies divided by their corresponding value for the mean model. It is seen from this figure that the probability density function of the first natural frequency of the constrained system closely match the distributions of the natural frequencies (divided by their corresponding value for the mean model) of the unconstrained system with the largest, i.e. 5.8\%, level of variations. Similarly, the distributions of the $2^{\text {nd }}-5^{\text {th }}$ natural frequencies of the constrained system (divided by their corresponding value for the mean model) match the similar probability density functions of all 5 natural frequencies of the unconstrained model with the lowest, i.e. $3.3 \%$, level of variations. It is thus concluded that the constrained model accurately matches the prescribed information on the variations of its natural frequencies.

\section{SUMMARY}

This paper focused on the modeling of structural uncertainty in dynamic systems through the randomization of the mass, stiffness, and/or damping matrices. Following a recently proposed strategy, the nonparametric methodology, the joint probability density function of the elements of the uncertain matrices are not specified but rather derived to achieve the maximum entropy which guarantees that "larger" deviations from the mean value will occur. The basic nonparametric formulation was revisited and validated on a new problem, i.e. the aeroelastic behavior of a aircraft wing. Recent extensions of the nonparametric methodology have also been briefly reviewed. In particular, it was shown that this methodology is applicable to geometrically nonlinear systems with minimal modifications from the original formulation. Finally, a constrained version of the nonparametric methodology was described that is particularly advantageous when accurate information regarding the system variability is available.

\section{REFERENCES}

Ghanem, R., and Spanos, P.D. 1991. Stochastic Finite elements: A spectral approach, Spring-Verlag, New York.

Kleiber, M., Tran, D.H., Hien, T.D. 1992. The Stochastic Finite element Method, John Wiley and Sons, new York, 1992.

Mignolet, M.P., Radu, A.G., and Gao, X., "Validation of Reduced Order Modeling for the Prediction of the Response and Fatigue Life of Panels Subjected to Thermo-Acoustic Effects," Proceedings of the 8th International Conference on Recent Advances in Structural Dynamics, Southampton, United Kingdom, Jul. 14-16, 2003.

Mignolet, M.P., and Soize, C., "Nonparametric Stochastic Modeling of Linear Systems with Prescribed Variance Information of Several Natural Frequencies," Proceeding of the Fifth International Conference On Computational Stochastic Mechanics, Rhodes, Greece, Jun. 21-23, 2006.

Mignolet, M.P., and Soize, C., "Stochastic Reduced Order Models for Uncertain Nonlinear Dynamical Systems," Proceedings of the International Modal Analysis Conference, IMAC XXV, Orlando, Florida, Feb. 19-22, 2007.

Radu, A., Yang, B., Kim, K., and Mignolet, M.P., "Prediction of the Dynamic Response and Fatigue Life of Panels Subjected to Thermo-Acoustic Loading," Proceedings of the 45th Structures, Structural Dynamics, and Materials Conference, Palm Springs, California, Apr. 19-22, 2004. Paper AIAA-2004-1557.

Rivas-Guerra, A.J., and Mignolet, M.P. 2004. Local/Global Effects of Mistuning on the Forced Response of Bladed Disks, Journal of Engineering for Gas Turbines and Power, Vol. 126, No. 1, pp. 131-141.

Schueller, G.I. (Ed.). 1997. A State-of-the-Art Report on Computational Stochastic Mechanics, Probabilistic Engineering Mechanics, Vol. 12, No. 4, pp. 197-321.

Soize, C. 2000. A nonparametric model of random uncertainties on reduced matrix model in structural dynamics, Probabilistic Engineering Mechanics, Vol. 15, No. 3, pp. 277-294. 
Soize, C. 2001. Maximum entropy approach for modeling random uncertainties in transient elastodynamics, Journal of the Acoustical Society of America, Vol. 109, No. 5, pp. 1979-1996.

Soize, C. 2005. Probabilistic models for computational stochastic mechanics and applications, Proceedings of the 9th International Conference on Structural Safety and Reliability ICOSSAR'05, Rome, Italy, 19-23 June 2005, Augusti, G., Schueller, G.I., and Ciampoli, M., (Eds). Millpress, Rotterdam, Netherlands, pp.23-42. 